\title{
Grouping Parallel Detection Method of UAV Based on Multi Features of Image Transmission Signal
}

\author{
Yuelei XIE ${ }^{1,2}$, Ping JIANG ${ }^{1,2}$, Xiao XIAO ${ }^{1,2}$ \\ ${ }^{1}$ School of Information and Communication, Guilin University of Electronic Technology, Guilin 541004, China \\ ${ }^{2}$ Ministry of Education Key Laboratory of Cognitive Radio and Information Processing, \\ Guilin University of Electronic Technology, Guilin 541004, China
}

879702235@qq.com,ylxie_guet@126.com,40438210@qq.com

Submitted February 5, 2021 / Accepted May 17, 2021

\begin{abstract}
The emergence of low, slow, and small civilian unmanned aerial vehicles (UAV) brings fun and convenience to life and work. However, with the widespread popularity of $U A V$, the illegal activities caused by them have gradually increased, causing great harm to social security. To solve this problem, in the paper, we propose a set of detection and recognition methods for UAV by UAV image transmission signal (ITS). The method is divided into two groups. In the first group, according to the signal characteristics in different transform domains such as spectrum and time-frequency spectrum, three sets of algorithms are proposed, which are time-frequency ridge double feature estimation (TFRDFE), segmented spectrum estimation (SSE) and cycle accumulation estimation of segmented spectrum (CAE-SS). Three sets of algorithms are estimated to perform blind detection on suspected UAV ITS. The second group uses the accurate recognition algorithm of UAV ITS to extract the periodic features in the signal, and completes the recognition of UAV through feature matching, decision criteria and other methods. The two groups of methods are implemented in parallel, and when the two groups both detect and recognize the flying target, it can be determined that there is UAV in the target airspace. The experimental results show that the recognition rate of the first group of suspected UAV ITS blind detection algorithm can reach $100 \%$ when the (signal-to-noise ratio) SNR is $-22 d B$. The second group of UAV ITS recognition algorithm can achieve $100 \%$ recognition rate when $S N R$ is $-4 \mathrm{~dB}$. Therefore, this method can complete the multi-target recognition of $U A V S$ and has practical application value.
\end{abstract}

\section{Keywords}

UAV, image transmission signal, time-frequency ridge double feature estimation, segmented spectrum estimation, cycle accumulation estimation of segmented spectrum, signal characteristics

\section{Introduction}

With the rapid development of information industry technology, the low, slow and small UAV industry has been developing rapidly, which attracts the attention of people all over the world [1], [2]. Because it adopts radio for signal control and has many characteristics such as low, slow and small, it is convenient to perform various tasks and provide many conveniences for life and work [3], [4].

Although consumer UAV brings a lot of convenience to all walks of life, the illegal use of consumer UAV also brings a series of harms to the society and the country. In recent years, it is often reported that UAV interferes with flights [5], [6]. In 2015, a small UAV that had crashed was found on the White House lawn [7]. The same year, a UAV invaded the Japanese Prime Minister's residence, carrying plastic containers containing liquid, which police believe may contain cesium released by Fukushima [8]. Not only that, criminals often use UAV cameras to illegally take pictures of military bases, major conferences, and other areas that involve personal privacy and national security [9-11]. The above actions not only violate the law, but also pose a serious threat to the national and social public security [12]. Therefore, it is extremely important to take a reasonable and effective control measure to prevent the illegal flight of UAV [13].

\section{Relative Work}

In order to reduce or eliminate the threat posed by the illegal flight of UAVs, many scientific researchers are studying the problem in a variety of ways, which are mainly reflected in three methods, radar detection [14], optical detection [15], [16], acoustics detection [17] and radio signal detection.

Radar detection is widely used in the detection field, and its effect is usually better [18]. However, the small size of UAV and low flight height, and the fact that plastic 
model is often used in the shell, affect the detection performance of radar. In addition, radar has the disadvantages of strong radiation, high power consumption and high cost.

Optical detection is a way to identify aerial targets through optical cameras [19]. Although this method has the effect of visual detection and recognition, it is limited by the external weather environment, resulting in a short detection distance.

Acoustic detection is a way to identify UAV by receiving sound signals emitted by UAV [20]. However, in urban environments, various vehicles usually generate more noise, and the rotor sound of the small UAV itself is relatively small, so this method is not suitable for longdistance detection.

Compared with the above optical, acoustic, radar detection, radio signal detection is not limited by the weather environment, and has many advantages such as low power consumption, no radiation and so on [21]. There are two ways of radio signal detection. One needs a thirdparty signal source. The third-party signal and the UAV rotor produce micro-Doppler effect as the identification method. The other is to intercept the UAV communication signal directly, and recognize it by analyzing the signal and extracting the signal features.

Reference [22] used two methods of radio signal detection to detect UAV, and achieved certain results, but there are also some problems. For example, the micro Doppler detection mode is limited by the flight attitude of UAV and relies too much on the nearby third-party signal source. The other is based on the periodicity of the ITS, which can be identified by extracting the periodic features. But in the actual test, the random signal nearby is easy to produce the same features, resulting in false alarm. Reference [23] also uses the periodicity of UAV signals to identify. In reference [24], the deep learning method is used to recognize the UAV communication signal, and the recognition rate is high. However, it has not been tested in low SNR, and compared with the traditional feature extraction engineering, the deep learning recognition method has higher complexity and hardware implementation difficulty.

The rest of this paper consists of the following parts: The third section describes the system architecture and signal model. The fourth section explains the blind detection of suspected UAV image transmission signal (BD-Suspected UAV ITS) and accurate recognition of UAV image transmission signal (AR-UAV ITS) algorithms in detail. In the fifth section, the BD-Suspected UAV ITS and AR-UAV ITS algorithms are simulated and verified. Finally, the sixth section gives the conclusion and prospect.

\section{Signal Model}

The civil UAV signals are mainly divided into two categories. One is the signal used by the remote control to control the UAV, that is the flight control signal [25]. The

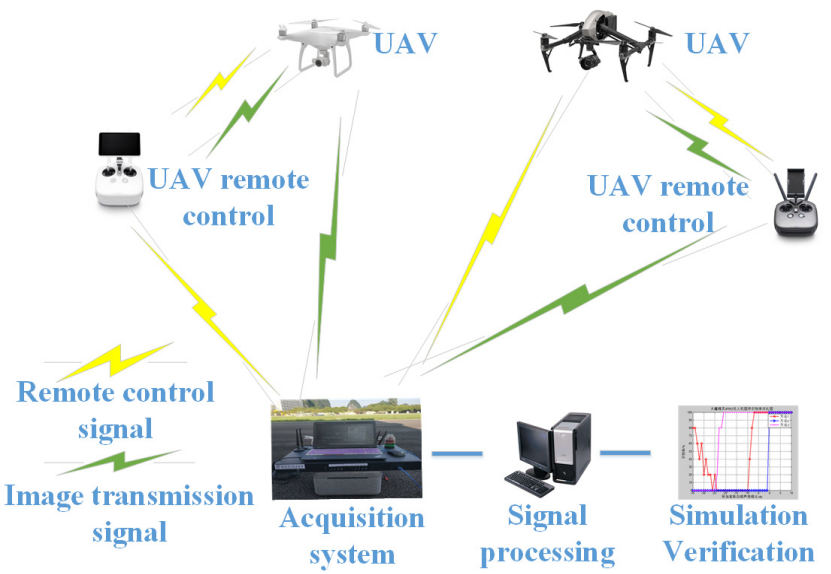

Fig. 1. UAV detection and identification deployment diagram.

other is the signal transmitted by the UAV to the remote control, that is the ITS. As shown in Fig. 1, the acquisition system can collect UAV flight control signals and ITS, and extract baseband signals through mixing, digital downconversion, and filtering. The baseband signals are processed by algorithms to complete the entire identification operation.

In this paper, the UAV ITSs are mainly studied, and OFDM communication is adopted for ITS [26-28]. OFDM is a multi-carrier modulation mode, which overcomes channel frequency selective fading by reducing and eliminating the influence of inter-symbol crosstalk. An OFDM symbol includes multiple modulated subcarriers. Assume that $N$ represents the number of subcarriers, and $T$ represents the duration of the OFDM symbol, $d_{i}(i=0,1, \ldots, N-1)$ is the data symbol allocated to each subcarrier, $f_{0}$ is the carrier frequency of the 0 -th subcarrier, Rectangular function $\operatorname{rect}(t)=1,|t| \leq T / 2$, then an OFDM symbol starting from $t=t_{\mathrm{s}}$ can be expressed as:

$$
\begin{aligned}
& s(t)= \\
& \begin{cases}\operatorname{Re}\left\{d_{i} \operatorname{rect}\left(t-t_{\mathrm{s}}-\frac{T}{2}\right) \exp \left[\mathrm{j} 2 \pi\left(f_{0}+\frac{i}{T}\right)\left(t-t_{\mathrm{s}}\right)\right]\right\} & , t_{\mathrm{s}} \leq t \leq\left(t_{\mathrm{s}}+T\right) \\
0 & , \text { others }\end{cases}
\end{aligned}
$$

where Re represents the real part data, $t_{\mathrm{s}}$ represents the sampling interval, and $\mathrm{j}$ is the imaginary unit.

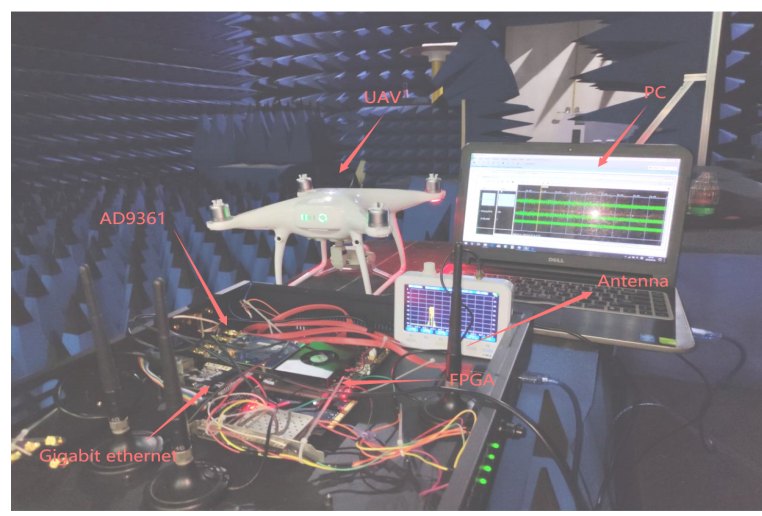

Fig. 2. Physical picture of acquisition system. 
In order to verify the signal model, the UAV ITS is collected by zero (intermediate frequency) IF receiver. The sampling rate of the acquisition system is 40 MSPS after down-conversion. The hardware diagram of the acquisition system is shown in Fig. 2.

Firstly, two DJI Phantom 4Pro UAVs, one DJI Inspire Two UAV and Wi-Fi signal are collected respectively, and the receiver acquisition time is $0.2 \mathrm{~s}$.

$$
X(t)=S(t)+N(t)
$$

where $t$ is the duration of $0.2 \mathrm{~s}, S(t)$ is the UAV ITS, $N(t)$ is the flight control signal, natural noise, radio interference signal, etc.

The signal is extracted by AD9361 Analog-digital conversion, mixing frequency, digital-analog down-conversion and bandpass filtering to obtain $12 \mathrm{bit}$ IQ signals.

$$
X(n)=S(n)+N(n) .
$$

Among them, $n=0,1, \ldots, 8 \times 10^{6}$, the Fast Fourier transform of the signal has the following evolution:

$$
X(K)=\sum_{n=0}^{N-1} X(n) w_{N}^{k n}, W_{N}=\exp \left(-\mathrm{j} \frac{2 \pi}{N}\right) .
$$

Among them, $K=0,1, \ldots, N-1, N=8 \times 10^{6}$.

Then, the time-frequency analysis of the measured signal is performed by segmented FFT, as shown in Fig. 3 .

It can be seen from the figure that the bandwidth of the UAV ITS and Wi-Fi signal is larger, and the duration time is longer in the time-frequency spectrum.

\section{UAV ITS Recognition Algorithm}

The UAV grouping parallel detection method based on the multi-features of the ITS is divided into two groups, each group is composed of different features, and the two groups are executed in parallel without affecting each other.

As shown in Fig. 4, the first group is algorithm A, which is used for BD-Suspected UAV ITS. Algorithm A is divided into three algorithms, namely TFRDFE, SSE and CAE-SS. The three algorithms are proposed in this paper through the signal characteristics in different transform domains such as frequency spectrum and time-frequency spectrum. The purpose is to find the optimal detection algorithm for suspected UAV.

The second group is algorithm $\mathrm{B}$, which is used for the AR-UAV ITS. Its core is to extract the specific characteristics of each UAV ITS through sliding shift cyclic autocorrelation feature extraction (SSCAFE) algorithm to identify the UAV.

Since the first group and the second group of algorithms run separately, they are prone to false alarms. Therefore, the two groups of algorithms adopt a parallel ope-

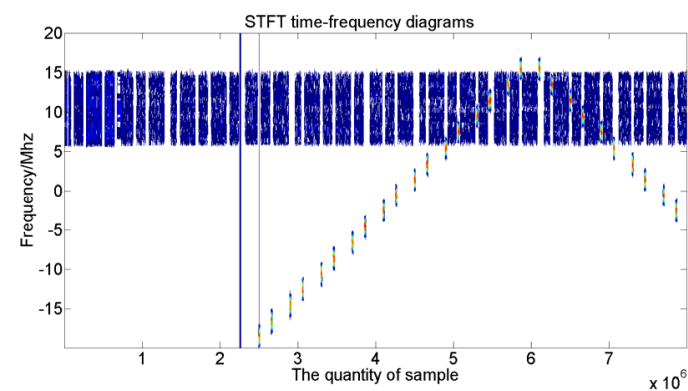

(a) DJI Phantom 4Pro Number One

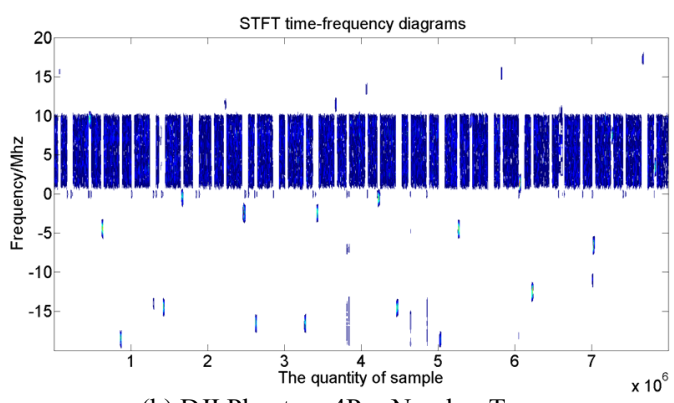

(b) DJI Phantom 4Pro Number Two

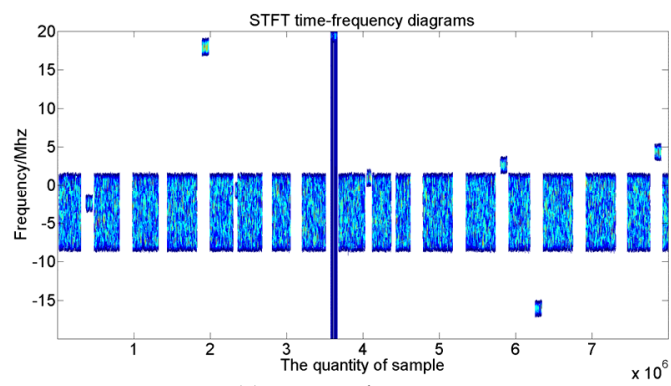

(c) DJI Inspire Two

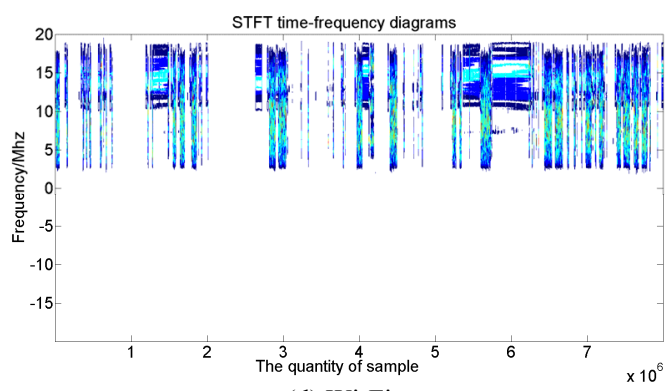

(d) $\mathrm{Wi}-\mathrm{Fi}$

Fig. 3. Spectrogram of the measured signal.

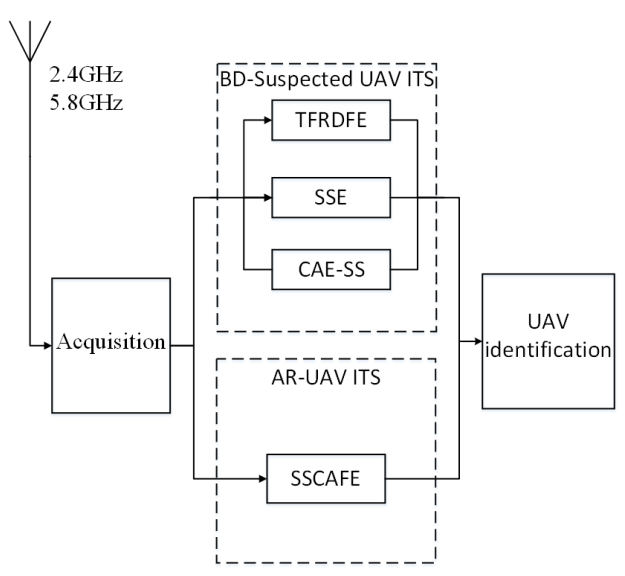

Fig. 4. Algorithm structure diagram. 
ration mode. When both groups detect and recognize the $\mathrm{UAV}$, it is deemed that the UAV exists.

\subsection{Algorithm A: BD-Suspected UAV ITS}

BD-Suspected UAV its method includes three sets of sub algorithms TFRDFE, SSE, CAE-SS, and all of them are proposed by the special characteristics of the signal transmitted in the frequency and time-frequency domain.

For the UAV ITS in frequency domain, the most typical characteristic is that it has a bandwidth of $10 \mathrm{MHz}$ and $20 \mathrm{MHz}$. Generally, only Wi-Fi and other multicarrier signals in ISM (Industrial Scientific Medical) band have this feature. Therefore, this paper proposes a UAV ITS detection and identification method based on signal bandwidth, such as SSE and CAE-SS.

For the time-frequency domain, because the time-frequency spectrum of UAV ITS has the characteristics of wide bandwidth and long duration time point, this paper proposes to extract the time-frequency ridge double features to detect the UAV ITS, such as TFRDFE. Among them, time-frequency analysis methods are widely used in many signal processing fields, including medical and biology [29], arc detection [30], signal estimation [31] and so on. This paper improves on the time-frequency analysis method, and proposes a set of time-frequency ridge dualfeature detection algorithm suitable for UAV detection.

In addition, the three proposed UAV detection methods under different domains and different characteristics will be explained one by one in this section. And in the next section, the three sets of methods will be compared by simulation, and other UAV detection methods will also be compared to select the optimal algorithm.

\subsubsection{TFRDFE Algorithm}

This algorithm mainly studies the extraction of UAV ITS characteristics in the time-frequency domain, and uses the characteristics to detect UAVs.

Step 1: Segmented signal processing:

Perform segmental FFT processing on the measured signal $x(t)$, a total of 7812 segments;

$$
X_{i}(n)=\sum_{i \times 1024}^{i \times 1024+1023} X\left(m_{i}\right) w_{1023}^{k n}
$$

Among them, $m_{i}=i \times 1024, i \times 1024+1, \cdots, i \times 1024+1023$; $n=0,1, \cdots 1023, k=0,1, \cdots 1023, i=0,1, \cdots 7812$.

Step 2: Time-frequency ridge line extraction:

The peak value and frequency value of each spectrum are extracted. Because each spectrum has a peak value, and 7812 spectrum has 7812 peak points, the peak points are connected one by one, which is the ridge line described in this paper.

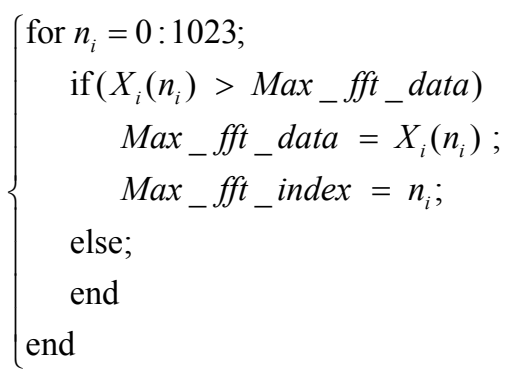

Among them, Max_fft_data is the maximum value of each segment and Max_fft_index is the maximum value coordinate.

\section{Step 3: Piecewise spectrum reconstruction:}

After extracting the time-frequency ridge line in the previous step, perform spectrum reconstruction to restore the true frequency value. When the ridge line value is greater than the noise threshold, the following algorithm steps are performed.

if $($ Max_fft_data $>$ threshold_fft_max $)$

$f f t_{-} f r e q u e n c y(i)=\left\{\begin{array}{l}\text { Max_fft_index }-512, M a x \_f f t \text { index } \geq 512 ; \\ \text { Max_fft_index }+512, \text { Max_fft_index }<512 ;\end{array}\right.$

else;

Among them, $i=0,1,2 \cdots 7812$.

Step 4: Interference elimination:

After the pretreatment of the previous step, the signal still contains various radio interference signals such as flight control signal, noise, $\mathrm{Wi}-\mathrm{Fi}$, etc. so most of the interference signals need to be removed to facilitate the subsequent algorithm analysis.

According to the time-frequency diagram, the nonITS is within $5 \mathrm{MHz}$ bandwidth, and the number of continuous time points is small. By analyzing the flight control signals and burst signals of multiple UAVs, it is concluded that the signal duration time threshold can be set to 100 , that is, less than 100 , and this segment of the signal is eliminated.

As shown in Fig. 5, it is the time-frequency diagram of DJI Phantom 4Pro Number One, which includes ITS, flight control signal and various electromagnetic interference signals. A flight control signal is gradually amplified and displayed, and its duration time points can be obtained through its left and right borders, which is approximately equal to 25000. Perform segmental FFT processing on the signal with a resolution of 1024 . Through calculation, the number of continuous segments of the flight control signal is approximately equal to 24 , and its value is much less than 100 , which satisfies the set conditions.

In addition, the acquisition range of each channel of the device is $40 \mathrm{MHz}$, and the ITS bandwidth of $10 \mathrm{MHz}$ or $20 \mathrm{MHz}$ is easily partly sampled by the first channel and 


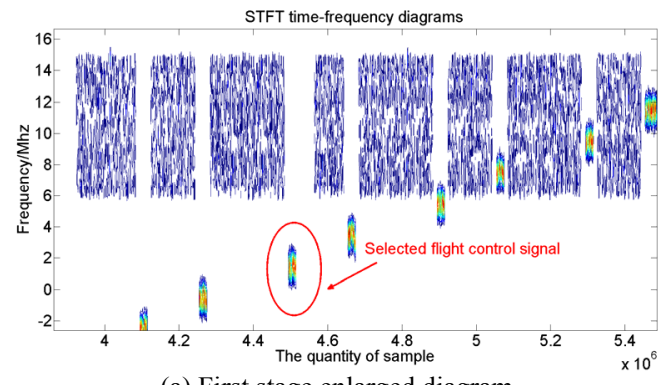

(a) First stage enlarged diagram

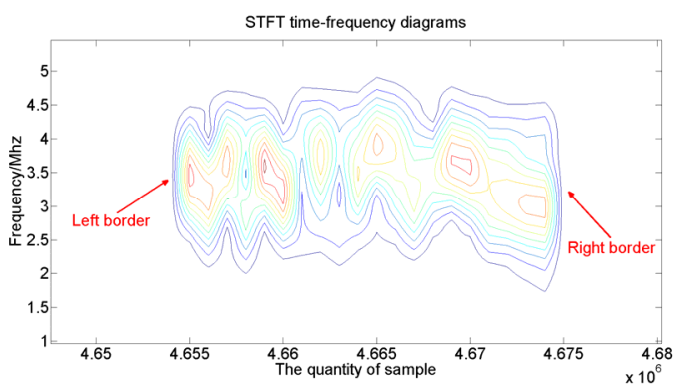

(b) Second stage enlarged diagram

Fig. 5. DJI Phantom 4 Pro Number one time-frequency diagram.

the other part is sampled by the second channel. For example, the first channel is sampled at $4 \mathrm{MHz}$, and the second channel is sampled at $6 \mathrm{MHz}$.

Therefore, $5 \mathrm{MHz}$ bandwidth and 100 duration time points can be set as the characteristic threshold to eliminate the interference signal. The details are as follows.

$c n t=$

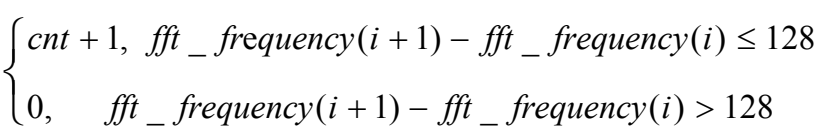

where $c n t$ is the number of duration time points.

In addition, by removing the ridge line value of each segment that does not meet the condition, the remaining time-frequency ridge line value segment $h$ will be less than or equal to 7812 .

Step 5: Bandwidth center coordinate estimation method:

Through the difference method, the first 100 times signal frequency duration time points within the $20 \mathrm{MHz}$ bandwidth of the ITS are counted and averaged. The average value can be assumed to be the center bandwidth point.

$$
\left\{\begin{array}{l}
\text { if }\left(\left(f f t \text { frequency }(h+1)-f f t_{-} f r e q u e n c y(h)\right) \leq 512\right) \\
\quad \text { band_est_cnt }=\text { band_est_cnt }+1 ; \\
\text { else } \\
\quad \text { band_est_cnt }=0 ;
\end{array}\right.
$$

Among them, band_est_cnt is the statistical value of the signal duration time point, and the value of $h$ is less than or equal to 7812 , which is the time-frequency ridge value segment after removing noise and interference signals.

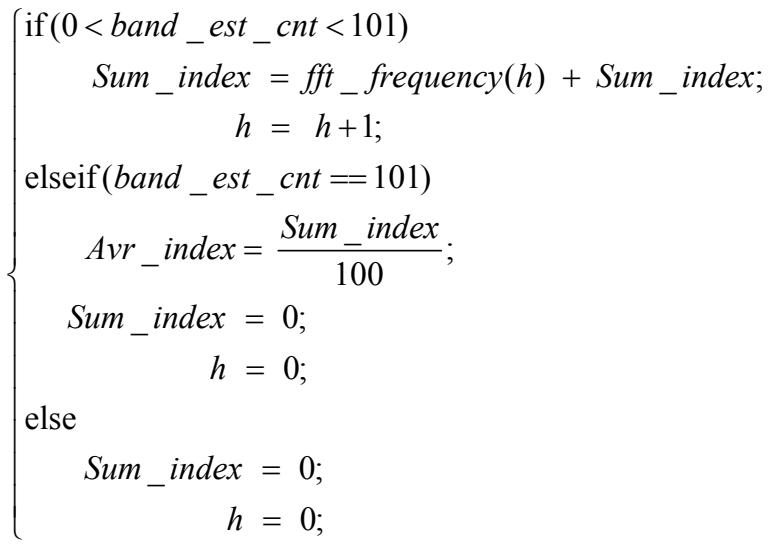

Among them, Sum_index is the sum of frequency points, and $A v r_{\text {index }}$ is its average value, which is the center bandwidth estimation point.

Step 6: Signal bandwidth estimation:

It is known that the maximum bandwidth of UAV ITS is $20 \mathrm{MHz}$, that is, the difference between all signal frequency points and its central bandwidth point is less than or equal to $10 \mathrm{MHz}$. According to this feature, by making a difference between the signal with interference removed and the central bandwidth point, when it is less than $10 \mathrm{MHz}$, the number of points after quantization is 256 , then counting.

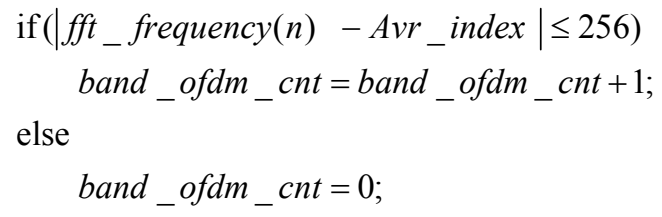

Among them, band_ofdm_cnt is the number of time duration time point, $n$ is less than or equal to 7812 .

It can be seen from Fig. 3 that the total number of continuous points of the UAV ITS in the time-frequency diagram is at least more than $1 / 4$, so it is defined as the characteristic of the time-frequency points of the ITS, and the threshold value is 1953 . If it is greater than this threshold, it is deemed that there is a suspected UAV ITS.

\subsubsection{SSE Algorithm}

The algorithm is mainly to transform the signal by segment spectrogram, and detect the signal according to the bandwidth value displayed in the spectrogram and the number of segments satisfying the bandwidth value. The algorithm is described in the following steps.

Step 1: Segmented signal processing:

This part is consistent with TFRDFE algorithm, and the segmented data is obtained.

Step 2: Segmented spectrum reconstruction:

First, it is necessary to satisfy that the maximum amplitude of the segment number is greater than the threshold before proceeding to the subsequent steps. Through 
this threshold, it can be judged whether there is a signal in the channel.

When $X_{i}(n)>$ threshold_fft_max, perform the following steps. $X_{i}(n)$ is the FFT amplitude value, $n$ is the frequency coordinate point, the resolution is 1024 .

The setting of the threshold determines the complexity of subsequent signal processing: The threshold is high and the complexity is low. Low threshold means high complexity, but farther detection distance;

$$
f f t_{-} \text {Amplitude }(n)= \begin{cases}X_{i}(n-512), & n \geq 512 \\ X_{i}(n+512), & n<512\end{cases}
$$

where fft_Amplitude is the spectrum amplitude value after spectrum reconstruction, $n$ is the number of frequency points, and the resolution is 1024 .

Step 3: Establishment of segmented spectrum threshold:

Analyze the 7812 segment of the spectrum, as shown in Fig. 6 for one of the segments, there are three different color threshold lines. After simulation verification, the green line is the best threshold line.

Step 4: Segment bandwidth statistics:

When the signal is larger than the threshold line, the number of consecutive points of the signal will be counted. If the signal is smaller than the threshold line, it will not be processed and the spectrum signal will be discarded. In fact, as shown in Fig. 6, the bandwidth of the ITS is $10 \mathrm{MHz}$, and each frequency point in the signal is not greater than the threshold line (green line), resulting in the wrong calculation of the signal bandwidth. The frequency points less than the threshold line can be called leakage points. In order to prevent the misjudgment of bandwidth caused by leakage, the leakage should be supplemented. After testing and verification, the leakage judgment threshold is set to 10 , that is, the spectrum amplitude of 10 consecutive points is allowed to be less than the threshold, which can better complete the bandwidth calculation of ITS.

$$
\left\{\begin{array}{l}
\text { if }(\text { del_cnt } \geq 10) \\
\quad \text { band_cnt }=0 \\
\text { elseif }(f f t \text { Amplitude }(n) \geq \text { band_threshold }) \\
\quad \text { band_cnt }=\text { band_cnt }+1 \\
\text { else; }
\end{array}\right.
$$

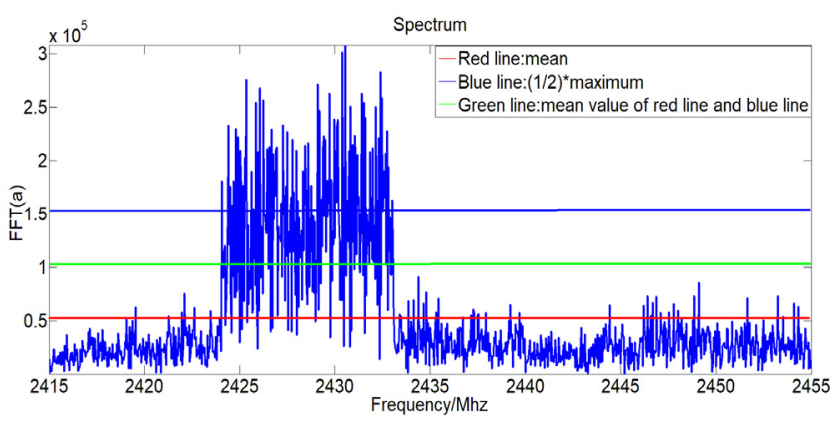

Fig. 6. DJI Phantom 4Pro Number One segmented spectrum.
From (13), when del_cnt is greater than or equal to 10 , band_cnt is reset to 0 . When del_cnt is less than 10 and fft_Amplitude $(n)$ is greater than or equal to band_threshold, band_cnt counts.

$$
\left\{\begin{array}{l}
\text { if }(\text { del_cnt } \geq 10) \\
\quad \text { del_cnt }=0 \\
\text { elseif }\left(f f t \_A m p l i t u d e(n) \geq \text { band_threshold }\right) \\
\quad \text { del_cnt }=0 \\
\text { elseif }(f f t \text { Amplitude }(n)<\text { band_threshold }) \\
\quad \text { del_cnt }=\text { del_cnt }+1 \\
\text { else; }
\end{array}\right.
$$

Equation (14) means that when del_cnt is greater than or equal to 10 , del_cnt is reset to 0 . When del_cnt is less than 10, if fft_Amplitude( $n$ ) is greater than or equal to band_threshold, del_cnt is reset to 0 , otherwise del_cnt continues to count. Where band_cnt is the bandwidth count value, del_cnt is the missing point count value, fft_Amplitude $(n)$ is the FFT amplitude, and band_threshold is the bandwidth threshold.

Similar to the TFRDFE algorithm, the number of segments of the entire signal must be at least $1 / 4$, that is, greater than or equal to 1953 , which can demonstrate that there is a suspected UAV ITS.

\subsubsection{CAE-SS Algorithm}

The CAE-SS algorithm is modified and optimized on the basis of the SSE algorithm.

\section{Step 1: Segmented signal processing:}

This part is consistent with the STFSE algorithm and the TFRDFE algorithm, and the segmented data $X_{i}(n)$ is obtained.

\section{Step 2: Segmented spectrum reconstruction:}

To reconstruct the frequency spectrum of the segmented signal, the formula is as follows.

$$
\begin{gathered}
f f t_{-} \text {Amplitude }_{0}(n)=\left\{\begin{array}{l}
X_{0}(n-512), n \geq 512 ; \\
X_{0}(n+512), n<512 ;
\end{array}\right. \\
f f t_{-} \text {Amplitude }_{1}(n)=\left\{\begin{array}{l}
X_{1}(n-512), n \geq 512 ; \\
X_{1}(n+512), n<512 ;
\end{array}\right. \\
\ldots \ldots . . \\
f f t_{-} \text {Amplitude }_{7812}(n)=\left\{\begin{array}{l}
X_{i}(n-512), n \geq 512 ; \\
X_{i}(n+512), n<512 ;
\end{array}\right.
\end{gathered}
$$

Among them, fft_Amplitude( $n$ ) is the segmented spectrum value, $n$ is the frequency value, ranging from 0 to 1023 . The segmented spectrum reconstruction diagram is in Fig. 7.

Step 3: Signal accumulation;

Perform one-to-one cumulative summation for each frequency point of each of the above signals, a total of 7812 times. 


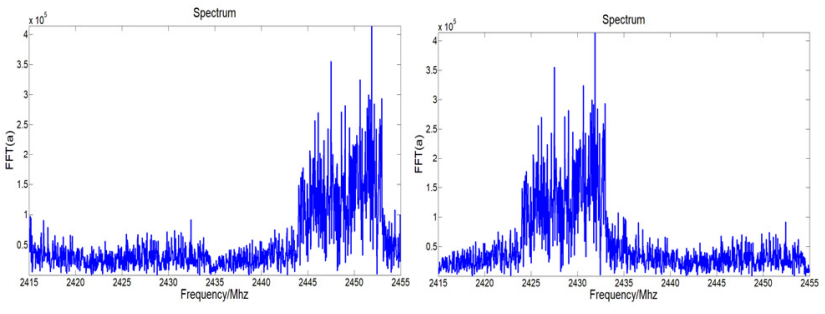

(a) Before spectrum reconstruction

Fig. 7. Segmented spectrum reconstruction diagram.

$$
\left\{\begin{array}{c}
\text { Sum_f }_{-} f t(0)=\sum_{i=0}^{n o c} f f t_{-} \text {Amplitude }_{i}(0) ; \\
\text { Sum_fft }_{-}(1)=\sum_{i=0}^{n o c} f f t_{-} \text {Amplitude }_{i}(1) ; \\
\ldots \ldots \\
\text { Sum_fft }(1023)=\sum_{i=0}^{n o c} f f t_{-} \text {Amplitude }_{i}(1023) ;
\end{array}\right.
$$

Among them, $i=0,1,2 \cdots, n o c$, noc $=7811$, Sum_fft $(i)$ is the cumulative sum.

Through accumulation, the signal amplitude increases and the spectral quality is obtained to optimize for easier signal detection. Figure 8 shows the cumulative spectrum of the measured signal after adding gaussian white noise. The noise is $20 \mathrm{~dB}$ and $-8 \mathrm{~dB}$, respectively.

\section{Step 4: Establishment of judgment threshold;}

As shown in Fig. 8, the blue line is the maximum, the red line is the average, and the green line is the average of the blue and red lines. Among them, the red threshold line and the green threshold line have the same effect in the case of $20 \mathrm{~dB}$, but the red threshold line is better under $-8 \mathrm{~dB}$. However, using the red line as the threshold is easy

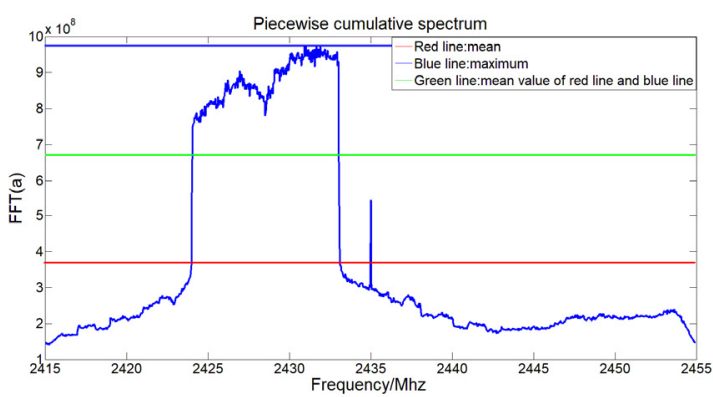

(a) $20 \mathrm{~dB}$ piecewise cumulative spectrum

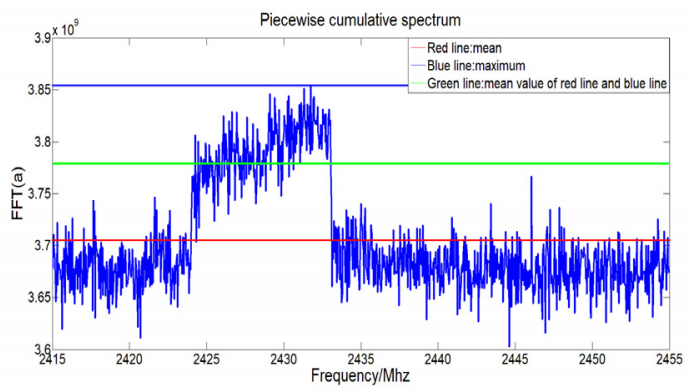

(b) $-8 \mathrm{~dB}$ piecewise cumulative spectrum

Fig. 8. Segmented spectrum reconstruction diagram.

to misjudge noise as a signal. Therefore, through experimental verification, setting the threshold of the number of missing points to 3 can prevent signal misjudgment and obtain the best recognition effect.

\section{Step 5: Signal judgment:}

This step is mainly used to determine whether the channel has a signal. Because there is only noise when there is no signal, there is no need to perform the next step of signal processing. The noise threshold can be set based on multiple actual collection statistics of the surrounding noise. When the spectrum accumulation signal is greater than the noise threshold, the next step is executed.

$$
\text { Sum_fft }(n)>\text { noise_threshold, } \quad n=0,1,2 \cdots 1023
$$

where noise_threshold is the set noise threshold.

\section{Step 6: Suspected OFDM signal estimation;}

Then, the bandwidth value is calculated by the missing point complementation algorithm, and the signal band_cnt is used to count the number of points continuously greater than the set signal judgment threshold (red threshold line) in the spectrogram. When the number of consecutive points meets the bandwidth greater than $5 \mathrm{MHz}$ and less than $20 \mathrm{MHz}$, it can be determined that there is a suspected image transmission signal. The details are as described in (18).

$$
\left\{\begin{array}{l}
\text { if }(\text { del_cnt } \geq 3) \\
\text { band_cnt }=0 \\
\text { del_cnt }=0 \\
\text { elseif }(\text { Sum_fft }(n) \geq \text { band_threshold }) \\
\text { band_cnt }=\text { band_cnt }+1 \\
\text { del_cnt }=0 \\
\text { elseif }(\text { Sum_fft }(n)<\text { band_thersold }) \\
\text { band_cnt }=\text { band_cnt; } \\
\text { del_cnt }=\text { del_cnt }+1 \\
\text { else; }
\end{array}\right.
$$

In the formula, the band_cnt and the del_cnt reset to 0 . if the del cnt is greater than or equal to 3 . When the del_cnt is less than 3 and the Sum_fft value is greater than or equal to the band_threshold, the band_cnt and the del_cnt reset to 0. When the del_cnt is less than 3 and Sum_fft the value is less than the band_threshold, the band_cnt count remains unchanged and the del_cnt count.

\begin{tabular}{|c|c|}
\hline Algorithm & Characteristic \\
\hline TFRDFE & $\begin{array}{c}\text { Time-frequency spectrum: } \\
\text { (1) Signal duration time point } \\
\text { (2) Signal bandwidth characteristics }\end{array}$ \\
\hline SSE & $\begin{array}{c}\text { Spectrum diagram: } \\
\text { (1) Signal bandwidth characteristics }\end{array}$ \\
CAE-SS & $\begin{array}{c}\text { (2) The number of segments that meet the bandwidth } \\
\text { requirements }\end{array}$ \\
\hline
\end{tabular}

Tab. 1. Characteristics comparison of sub algorithms for BD- suspected UAV ITS. 
Here del_cnt is the leakage point meter value, band_cnt is the bandwidth meter value, Sum_fft is the integral amplitude of FFT, band_threshold is the bandwidth threshold. When the bandwidth points meet the requirements in the range of $5 \mathrm{MHz}$ to $20 \mathrm{MHz}$, it can be regarded as a suspected UAV ITS.

For the BD-suspected UAV ITS, the signal characteristics of the three sub algorithms included in the algorithm are shown in Tab. 1.

\subsection{Algorithm B: AR-UAV ITS}

UAV ITS belongs to OFDM signal, and OFDM signal is composed of cyclic prefix and useful signal length. The structure of OFDM symbol is shown by Fig. 9. Therefore, the total data length of one OFDM symbol is the sum of preamble data length and useful data length.

Cyclic prefix of each symbol is performed as guard interval with copying the last part of signal data to the front of the symbol. For OFDM signal with cyclic prefix, cyclostationary characteristics could be detected on each frame symbol of the signal [32]. The mathematical description is that the autocorrelation function of OFDM signal is a periodic function of time. It is learned that OFDM technique with cyclic prefix is widely used in the downlink of UAVs. Thus, features with cyclostaionary characteristics could be helpful to detect and identify UAV signals.

However, OFDM signal transmission mechanisms of different devices usually have different useful symbol length, so according to this characteristic, it can be used to distinguish different types of UAVs. Therefore, the algorithm is also defined as the AR-UAV ITS, which is mainly composed of SSCAFE algorithm, decision criteria and other signal processing methods. The algorithm is described in detail step by step as follows:

Step 1: Sliding shift cyclic autocorrelation:

If signal $s(t)$ is a cyclically stationary signal, the following two formulas hold.

$$
\begin{gathered}
m_{\mathrm{S}}(t)=E[s(t)]=m_{\mathrm{S}}\left(t+T_{0}\right), \\
R_{\mathrm{S}}(t, \tau)=\mathrm{E}\left\{\mathrm{s}(t+\tau / 2) s^{*}(t-\tau / 2)\right\}=R_{\mathrm{S}}\left(t+T_{0}, \tau\right) .
\end{gathered}
$$

It is said that the signal $s(t)$ is a cyclostationary signal with $T_{0}$ as the cycle period.

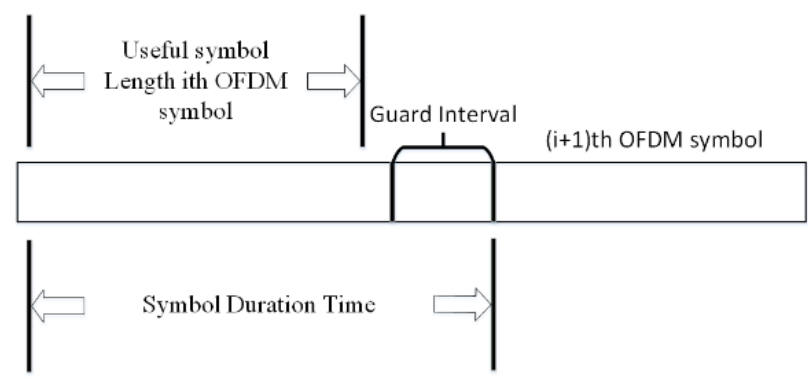

Fig. 9. OFDM symbol structure.
Obviously, the expansion of time-varying autocorrelation function $R_{\mathrm{S}}(t, \tau)$ can be written into the expression of Fourier series according to the Fourier expansion formula of periodic signal as shown in the following formula.

$$
R_{\mathrm{S}}(t, \tau)=\sum_{m=-\infty}^{\infty} R_{\mathrm{S}}^{\alpha} \mathrm{e}^{\mathrm{j} 2 \pi m t / T_{0}}=\sum_{m=-\infty}^{\infty} R_{\mathrm{S}}^{\alpha} \mathrm{e}^{\mathrm{j} 2 \pi \alpha t}
$$

where $\alpha=m / T_{0} . \quad R_{\mathrm{S}}{ }^{\alpha}(\tau)$ represents the Fourier series coefficient of the periodic correlation function $R_{\mathrm{S}}(t, \tau)$ and the following formula can be obtained.

$$
R_{\mathrm{S}}^{\alpha}(\tau)=\frac{1}{T_{0}} \int R_{\mathrm{S}}(t, \tau) \mathrm{e}^{-\mathrm{j} 2 \pi \alpha t} \mathrm{~d} t
$$

The Fourier series coefficient $R_{\mathrm{S}}{ }^{\alpha}(\tau)$ is called the cyclic autocorrelation function. According to the principle of signal sampling, the sampling period is $T_{0}$, sampling at any time has ergodic property of all states, so the mean value of the sampling sample can be used to estimate the autocorrelation function value $R_{\mathrm{S}}(t, \tau)$.

$$
R_{\mathrm{S}}(t, \tau)=\lim _{N \rightarrow \infty} \frac{1}{2 N+1} \sum_{n=-N}^{N} S\left(t+n T_{0}\right) S^{*}\left(t+n T_{0}-\tau\right)
$$

Substitute $R_{\mathrm{S}}(t, \tau)$ into (22), and the following equation can be obtained:

$$
R_{\mathrm{S}}^{\alpha}(\tau)=\lim _{N \rightarrow \infty} \frac{1}{2 N+1} \frac{1}{T_{0}} \int_{-T_{0} / 2-N T_{0}}^{T_{0} / 2+N T_{0}}\left(s(t) s^{*}(t-\tau) \mathrm{e}^{-\mathrm{j} 2 \pi \alpha t} \mathrm{~d} t\right)
$$

When $T=(2 N+1) T_{0}$ is set, we can get:

$$
R_{\mathrm{S}}^{\alpha \stackrel{T=(2 N+1) T_{0}}{=}} \lim _{T \rightarrow \infty} \frac{1}{T} \int_{-T / 2}^{T / 2} s(t) s^{*}(t-\tau) \mathrm{e}^{-\mathrm{j} 2 \pi \alpha t} \mathrm{~d} t
$$

where $\alpha$ is the cycle frequency.

Discrete sampling of the above formula can get the following formula:

$$
R_{\mathrm{S}}^{\alpha}(m)=\frac{1}{N} \sum_{n=0}^{N-1} s[n] s^{*}[n-m] \mathrm{e}^{-\mathrm{j} 2 \pi \alpha n} .
$$

In the three-dimensional diagram of OFDM cycle autocorrelation function $R_{\mathrm{S}}{ }^{\alpha}(\tau)$, when $\alpha$ is 0 and $\tau$ is equal to the useful signal length of OFDM, the peak value will appear. Therefore, according to each UAV ITS has different useful signal duration length, it is used as a characteristic value for UAV ITS recognition.

\section{Step 2: Establish a feature library:}

According to the ITS of each model of UAV has different useful signal length, it is used as a feature for UAV identification. The feature library is extracted from multiple UAVs by SSCAFE algorithm in advance.

\section{Step 3: Signal recognition:}

SSCAFE algorithm is used to extract the feature of the test target signal, and the feature is matched with the feature library. The matching method needs to establish a judgment criterion, which is as follows: 
Repeat steps one to three of the SSCAFE algorithm ten times. If three consecutive adjacent features are the same as the signal feature library of a certain model of $\mathrm{UAV}$, it can be regarded as that model of UAV. If three consecutive adjacent segments have the same characteristic but not the same as the UAV ITS feature library, and are not the same as the Wi-Fi signal feature library, they can be identified as an unknown suspected UAV. This criterion is based on actual verification and can prevent signal contingency.

\section{Simulation}

\subsection{Experimental test A: BD-Suspected UAV ITS}

In this experiment, two DJI Phantom 4Pro UAVs, one DJI Inspire Two UAV and Wi-Fi signals were tested. The signal is collected by the zero IF receiver with a sampling rate of 40 MSPS.

Experiment 1: The three algorithms of BD-Suspected UAV ITS, TFRDFE, SSE, and CAE-SS, are used to test, and the recognition rate reached $100 \%$. The measured signal is collected at a short distance, but in practice, the UAV has a longer distance and higher altitude from the protected core area. Theoretically speaking, the farther and higher the flying target that can be identified, the better is the effect of the recognition algorithm. For this reason, in the simulation experiment, we superimpose Gaussian random white noise on the measured signal to reduce the SNR, thereby verifying the anti-noise performance of the system. The specific experiment is as described in Experiment 2 .

Experiment 2: On the basis of the original measured data, Gaussian random white noise is superimposed to obtain a graph with the SNR as the horizontal axis and the recognition rate as the vertical axis, which effectively proves the respective performance of the three algorithms TFRDFE, SSE, and CAE-SS. The time-frequency diagram of signal superimposed with $0 \mathrm{~dB}$ noise is in Fig. 10.

It can be seen from Fig. 10 that there are still UAV ITS at $5 \mathrm{MHz}$ to $15 \mathrm{MHz}$, but under the noise flood, the time-frequency spectrum of the ITS becomes dim. In addition, the UAV remote control signal can be seen in the spectrum, which can also be used for UAV identification, but this article has not studied the signal at present. Therefore, it can be concluded that after Gaussian random white noise is superimposed, the entire time-frequency diagram is full of noise interference points, and the signal quality is reduced. As the SNR decreases, its performance impact is greater.

In this experiment, the recognition rate of the DJI Phantom 4Pro Number One, DJI Phantom 4Pro Number Two, DJI Inspire Two, and Wi-Fi signal under SNR of $-30 \mathrm{~dB}$ to $5 \mathrm{~dB}$ was analyzed. The recognition rate curve is in Fig. 11.

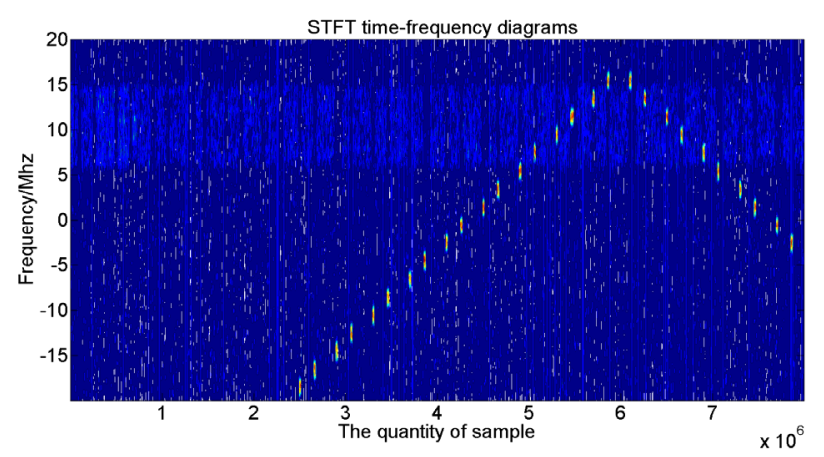

Fig. 10. Time-frequency analysis diagram of signal with signalto-noise ratio of $0 \mathrm{~dB}$.

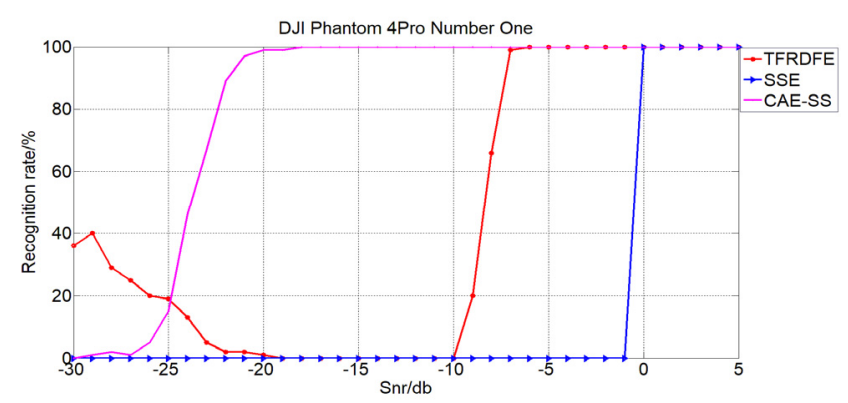

(a) DJI Phantom 4Pro Number One

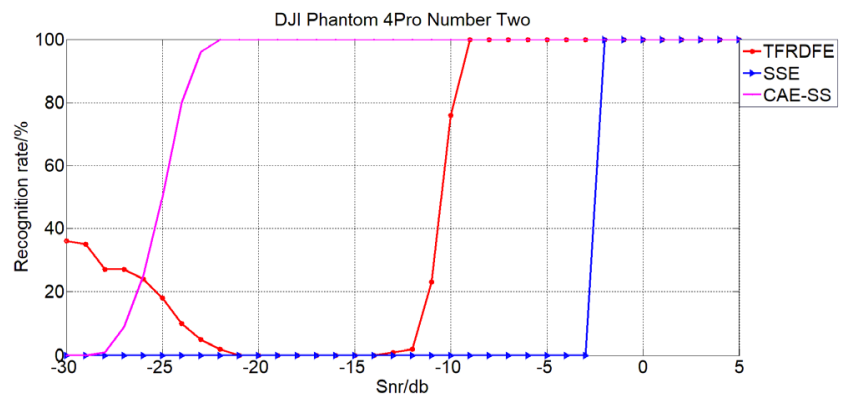

(b) DJI Phantom 4Pro Number Two

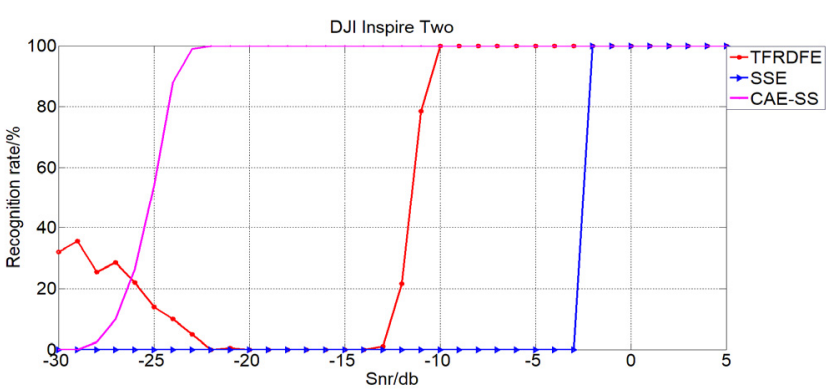

(c) DJI Inspire Two

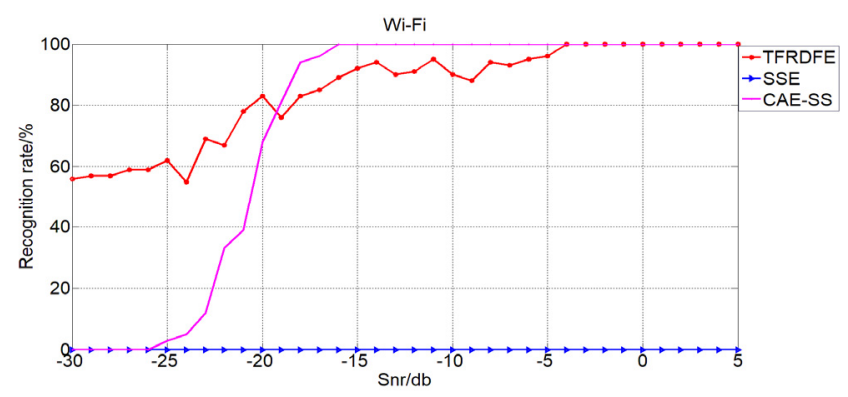

(d) Wi-Fi

Fig. 11. Comparison of the recognition rate curves of BDsuspected UAV ITS. 
According to Fig. 11(a) DJI Phantom 4Pro Number One recognition rate curve simulation, the TFRDFE algorithm drops to $0 \%$ at $-10 \mathrm{~dB}$, but starts to fluctuate at $-21 \mathrm{~dB}$. This is because at $-21 \mathrm{~dB}$, the noise is too large, the time-frequency spectrum is full of noise, and the timefrequency characteristics are changed, resulting in the algorithm error recognition. Therefore, when it is less than $-21 \mathrm{~dB}$, the recognition rate of the algorithm is wrong. As can be seen from Fig. 11(a), the SSE algorithm has a recognition rate of $0 \%$ at $-1 \mathrm{~dB}$, and then it has been $0 \%$. It can be seen from Fig. 11(a) that the CAE-SS algorithm decreases when the SNR is $-18 \mathrm{~dB}$, until $-30 \mathrm{~dB}$ it is $0 \%$. Through analysis, the CAE-SS algorithm has the best performance for the DJI Phantom 4Pro Number One, followed by the TFRDFE algorithm, but the TFRDFE algorithm is prone to misjudge in a low SNR environment, leading to false alarms. For DJI Phantom 4Pro Number Two and DJI Inspire Two, the recognition rate curve characteristics are similar to DJI Phantom 4Pro Number One.

Moreover, CAE-SS algorithm can meet $100 \%$ recognition rate of UAV at $-22 \mathrm{~dB}$, TFRDFE algorithm can meet $100 \%$ recognition rate of UAV at $-10 \mathrm{~dB}$. And they are superior to the UAV detection and recognition method based on micro-Doppler proposed in [23], and the UAV recognition method based on Convolutional Neural Networks and deep learning in [24] and [33].

In addition, for BD-Suspected UAV ITS, the performance comparison of the three sets of algorithms proposed in this paper can be shown in Tab. 2 .

As can be seen from Tab. 2 and the above simulation diagram: For the algorithm recognition performance, CAESS $>$ TFRDFE $>$ SSE. For algorithm complexity, TFRDFE $>$ CAE-SS $>$ SSE. In summary, the CAE-SS is the optimal algorithm of the first group.

\subsection{Experimental test B: AR-UAV ITS}

Although the performance of the first group of three suspected ITS detection algorithms is verified by experiments, the algorithms can effectively complete the target detection. But in fact, the bandwidth characteristics of the first group of algorithms cannot distinguish between $\mathrm{Wi}-\mathrm{Fi}$ and UAV, and it is easy to give false alarms only through BD-suspected UAV ITS. In response to the above problems, the AR-UAV ITS algorithm is used to distinguish between UAVs and Wi-Fi. The specific experiment is as follows:

Experiment 1: AR-UAV ITS algorithm is composed of SSCAFE algorithm and decision criteria. In the experiment, the ITS and Wi-Fi signal (analog interference signal) of three UAVs were simulated, and the sliding shift cyclic autocorrelation diagram was obtained.

Through the sliding shift cyclic autocorrelation diagram of the above four kinds of measured signals, as shown in Fig. 12, the abscissa is the number of delay points, and the ordinate is the data amplitude. The above characteristic results can be shown in Tab. 3.

\begin{tabular}{|c|l|}
\hline Algorithm & \multicolumn{1}{|c|}{ Advantages and disadvantages } \\
\hline TFRDFE & (1) Slightly poor noise immunity \\
& (2) Unstable performance \\
& (3) High complexity \\
\hline SSE & (1) Poor noise immunity \\
& (2) Stable performance \\
& (3) Low complexity \\
\hline CAE-SS & (1) Strong anti-noise performance \\
& (2) Stable performance \\
& (3) Slightly higher complexity \\
\hline
\end{tabular}

Tab. 2. BD-suspected UAV ITS performance comparison table.

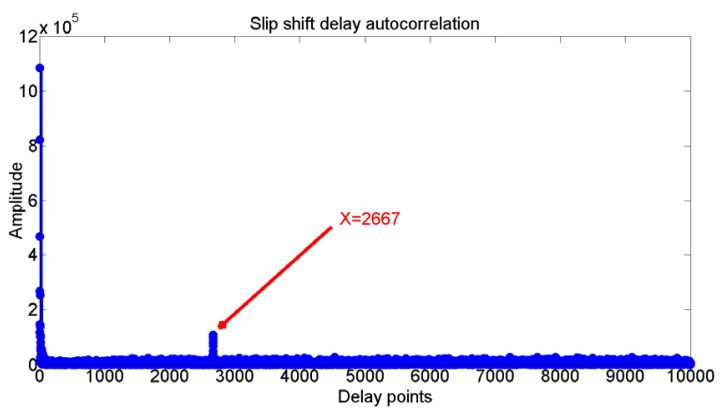

(a) DJI Phantom 4Pro Number One

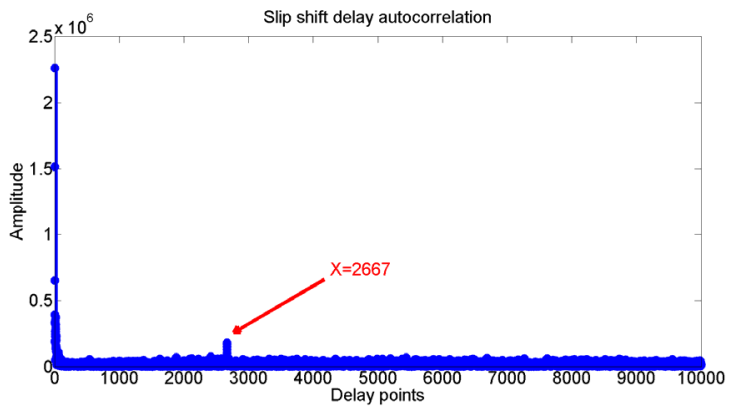

(b) DJI Phantom 4Pro Number Two

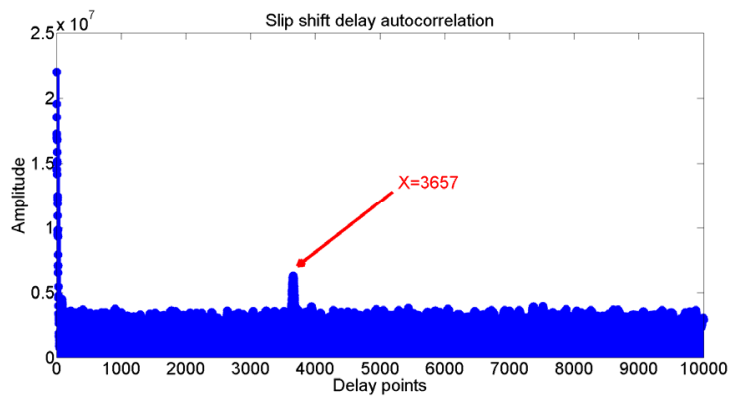

(c) DJI Inspire Two

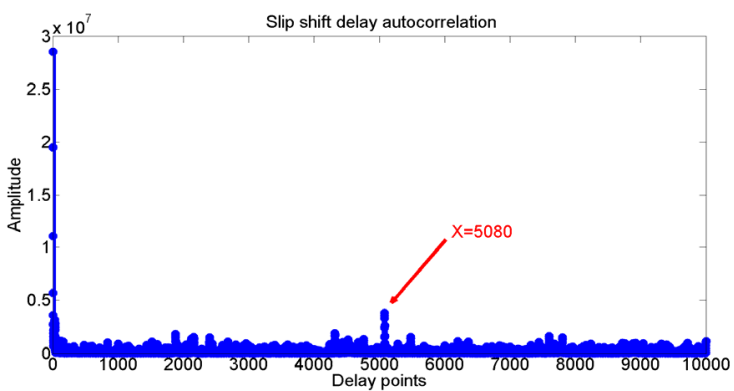

(d) Wi-Fi

Fig. 12. Sliding shift cyclic autocorrelation diagram of ITS 


\begin{tabular}{|l|c|}
\hline \multicolumn{1}{|c|}{ Test target } & $\begin{array}{c}\text { Signal delay points } \\
\text { (characteristic value) }\end{array}$ \\
\hline DJ Phantom 4PRO Number One & 2667 \\
\hline DJ Phantom 4PRO Number Two & 2667 \\
\hline DJ Inspire Two & 3657 \\
\hline Wi-Fi & 5080 \\
\hline
\end{tabular}

Tab. 3. Test signal sliding shift cyclic autocorrelation characteristic value.

It can be seen from Fig. 12 and Tab. 3 that different types of UAVs and Wi-Fi signals have different sliding shift cyclic autocorrelation characteristics, and the same type of UAVs have the same characteristics.

Experiment 2: In order to better verify the recognition performance of the algorithm, Random Gaussian white noise was superimposed on the basis of experiment 1 , and the above three types of UAV ITS are further classified and recognized. The recognition rate curve is shown in Fig. 13.

In Fig. 13 the abscissa is the SNR and the ordinate is the recognition rate. According to the analysis in Fig. 13, the recognition rate of DJI Inspire Two reaches $100 \%$ at $-4 \mathrm{~dB}$. With the decrease of SNR, the recognition rate gradually decreases to $0 \%$ at $-12 \mathrm{~dB}$. The recognition rate of DJI Phantom 4Pro Number One reach $100 \%$ at $-2 \mathrm{~dB}$ and decrease to $0 \%$ at $-10 \mathrm{~dB}$ with the SNR decreasing. The recognition rate of DJI Phantom 4Pro Number Two is $100 \%$ at $2 \mathrm{db}$ and $0 \%$ at $-8 \mathrm{~dB}$. In Fig. 13, the recognition rate of the three UAVs decreases with the decrease of SNR, among which the recognition rate of DJI Inspire Two is the strongest.

Because the quality of signal collected by each UAV is different, and the actual test signal itself is not pure signal. Therefore, the recognition rate of the same type of UAV is slightly different. However, through analysis, the recognition rate of $\mathrm{UAV}$ is almost $0 \%$ at $-12 \mathrm{~dB}$ and below, and almost $100 \%$ at $4 \mathrm{~dB}$ and above. Compared with the BD-suspected UAV ITS, the algorithm is relatively poor in the case of low SNR.

In general, the AR-UAV ITS can distinguish the UAV type, and can also identify whether there is UAV ITS from Wi-Fi signal.

In addition, compared with the algorithm based on convolutional neural network proposed in references [24] and [33], AR-UAV ITS algorithm has lower complexity and higher recognition rate. Therefore, the algorithm can be used as an effective method for UAV detection and recognition.

\section{Conclusion}

In this paper, we propose a parallel detection method for UAV group based on multi characteristics of ITS. The method is divided into two groups, the first group is used for BD-suspected UAV ITS, and the second group is used for AR-UAV ITS. In order to improve the efficiency of the

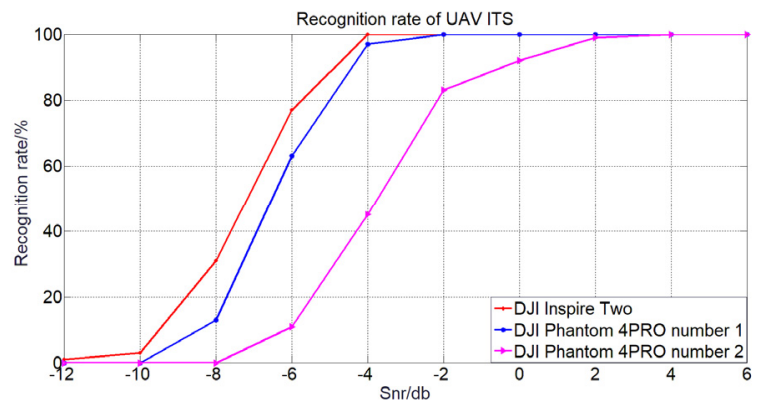

Fig. 13. AR-UAV ITS recognition rate curve.

\begin{tabular}{|c|l|}
\hline Algorithm & \multicolumn{1}{|c|}{ Advantages and disadvantages } \\
\hline & (1) Strong anti-noise performance \\
First group & (2) Low complexity \\
(BD-Suspected & (3) Unable to distinguish between UAV image \\
UAV ITS) & $\begin{array}{l}\text { transmission signal and other multi-carrier } \\
\text { signals }\end{array}$ \\
\hline & (1) The specific UAV model can be identified \\
Second Group & (2) Multi-target recognition \\
(AR-UAV ITS) & (3) Poor noise immunity \\
& (4) High complexity \\
\hline
\end{tabular}

Tab. 4. Comparison of the advantages and disadvantages of the two groups of algorithms.

algorithm, parallel implementation is adopted. For the BDsuspected UAV ITS algorithm, including TFRDFE, SSE, CAE-SS three algorithms are all proposed in the research process of this article. According to the experimental test, CAE-SS has the best performance, and the recognition rate can reach $100 \%$ when the SNR is $-22 \mathrm{~dB}$. For AR-UAV ITS, it is composed of SSCAFE algorithm and decision criteria. The experimental results show that the recognition rate of the algorithm reaches $100 \%$ when the SNR is $-4 \mathrm{~dB}$. The advantages and disadvantages of the two groups of algorithms can be shown in Tab. 4 .

It can be seen from Tab. 4 that the suspected UAV ITS can be judged by the BD-suspected UAV ITS algorithm, but it is also easy to misidentify the remaining multicarrier signals. The AR-UAV ITS algorithm can distinguish different multi-carrier signals, thereby identifying which UAV the signal belongs to, but the algorithm is easy to misidentify when the SNR is low. Because of the two groups of algorithms, the BD-suspected UAV ITS algorithm has strong anti-noise performance, but does not have the function of UAV type recognition, while the AR-UAV ITS algorithm has the function of UAV type recognition, but the anti-noise performance is poor, easy to misjudge. Therefore, in order to ensure the stability of the recognition algorithm, the two groups of algorithms are combined to make up for their shortcomings and improve the detection and recognition performance.

At present, there are many researches on UAV identification, but there are relatively few researches on UAV identification by wireless signal, especially by using the radio signal detection method and realized by the specific underlying hardware. Therefore, in the follow-up research, we need to optimize the algorithm and use hardware to realize the whole system, complete the system development, and improve the practical value of the algorithm. 


\section{Acknowledgments}

This work was supported by the Major Special Driven Projects in Guangxi Province, China, under Grant AA17202022; the Innovation Project of Guangxi Graduate Education, China, under Grant 2020YCXS021; the GUET Excellent Graduate Thesis Program, China, under Grant $18 Y J P Y S S 07$.

\section{References}

[1] PAUlSON, C. A., SOBESTER, A., SCANLAN, J. P. The rapid development of bespoke small unmanned aircraft: A proposed design loop. Aeronautical Journal, 2017, vol. 121, no. 1245, p. 1683-1710. DOI: 10.1017/aer.2017.99

[2] FLOREANO, D., WOOD, R. J. Science, technology and the future of small autonomous drones. Nature, 2015, vol. 521, no. 7553, p. 460-466. DOI: $10.1038 /$ nature 14542

[3] SHIELDS, B. Putting drones to work for water. Public Works, 2018, vol. 149, no. 1, p. 31-32.

[4] ESTRADA, M. A. R. The uses of drones in case of massive epidemics contagious diseases relief humanitarian aid: WuhanCOVID-19 crisis. SSRN Electronic Journal, 2020. DOI: $10.2139 / \mathrm{ssrn} .3546547$

[5] LI, N. Drone invasion of the airport and its police control. Journal of Jiangsu Police Institute, 2019, vol. 34, no. 3, p. 93-99. (in Chinese)

[6] SANTAMARINA CAMPOS, V. European Union policies and civil drones. In de Miguel Molina, M., Santamarina Campos, V. (eds.) Ethics and Civil Drones. Springer Briefs in Law. Cham: Springer, 2018. P. 35-41. DOI: 10.1007/978-3-319-71087-7_3

[7] SCHROEDER, R. Obama: Drone That Landed at White House for Sale at Radio Shack. [online] Available at: https://www.marketwatch.com/story/obama-drone-that-landed-atwhite-house-for-sale-at-radio-shack-2015-01-27.

[8] FACKLER, M. Drone, possibly radioactive, unsettles Japan. New York Times, 2015.

[9] ALTAWY, R., YOUSSEF, A. M. Security, privacy, and safety aspects of civilian drones: a survey. ACM Transactions on CyberPhysical Systems, 2017, vol. 1, no. 2, p. 1-25. DOI: $10.1145 / 3001836$

[10] PUTTOCK, A. K., CUNLIFFE, A. M., ANDERSON, K., et al. Aerial photography collected with a multirotor drone reveals impact of Eurasian beaver reintroduction on ecosystem structure. Journal of Unmanned Vehicle Systems, 2015, vol. 3, no. 3, p. 123-130. DOI: 10.1139/juvs-2015-0005

[11] WANG Y., XIA, H., YAO, Y., et al. Flying eyes and hidden controllers: A qualitative study of people's privacy perceptions of civilian drones in the US. Proceedings on Privacy Enhancing Technologies, 2016, vol. 2016, no. 3, p. 172-190. DOI: $10.1515 /$ popets-2016-0022

[12] LIN, C., HE, D., KUMAR, N., et al. Security and privacy for the internet of drones: Challenges and solutions. IEEE Communications Magazine, 2018, vol. 56, no. 1, p. 64-69. DOI: 10.1109/MCOM.2017.1700390

[13] WATKINS, S., BURRY, J., MOHAMED, A., et al. Ten questions concerning the use of drones in urban environments. Building and Environment, 2020, vol. 167, p. 1-10. DOI: 10.1016/j.buildenv.2019.106458
[14] DROZDOWICZ, J., WIELGO, M., SAMCZYNSKI, P., et al. $35 \mathrm{GHz}$ FMCW drone detection system. In Proc. of the 17th International Radar Symposium (IRS). Krakow (Poland), 2016, p. 1-4. DOI: 10.1109/IRS.2016.7497351

[15] BUSSET, J., PERRODIN, F., WELliG, P., et al. Detection and tracking of drones using advanced acoustic cameras. In Proc. SPIE 9647, Unmanned/Unattended Sensors and Sensor Networks XI; and Advanced Free-Space Optical Communication Techniques and Applications, Oct. 2015, p. 1-8. DOI: 10.1117/12.2194309

[16] KIM, B. H., KHAN, D., BOHAK, C., et al. V-RBNN based small drone detection in augmented datasets for 3D LADAR system. Sensors, 2018, vol. 18, no. 11, p. 1-16. DOI: $10.3390 / \mathrm{s} 18113825$

[17] BERNARDINI, A., MANGIATORDI, F., PALLOTTI, E., et al. Drone detection by acoustic signature identification. Electronic Imaging, 2017, vol. 2017, no. 10, p. 60-64. DOI: 10.2352/ISSN.2470-1173.2017.10.IMAWM-168

[18] SHIN, D., JUNG, D., KIM, D., et al. A distributed FMCW radar system based on fiber-optic links for small drone detection. IEEE Transactions on Instrumentation and Measurement, 2017, vol. 66, no. 2, p. 340-347. DOI: 10.1109/TIM.2016.2626038

[19] HAMMER, M., HEBEL, M., LAURENZIS, M., et al. Lidar-based detection and tracking of small UAVs. In Proceedings Volume 10799, Emerging Imaging and Sensing Technologies for Security and Defence III; and Unmanned Sensors, Systems, and Countermeasures. 2018, p. 1-9. DOI: 10.1117/12.2325702

[20] YUE, X., LIU, Y., WANG, J., et al. Software defined radio and wireless acoustic networking for amateur drone surveillance. IEEE Communications Magazine, 2018, vol. 56, no. 4, p. 90-97. DOI: 10.1109/MCOM.2018.1700423

[21] NGUYEN, P., RAVINDRANATHA, M., NGUYEN, A., et al. Investigating cost-effective RF-based detection of drones. In DroNet '16: Proceedings of the 2nd Workshop on Micro Aerial Vehicle Networks, Systems, and Applications for Civilian Use. 2016, p. 17-22. DOI: 10.1145/2935620.2935632

[22] HUA, F., ABEYWICKRAMA, S., ZHANG, L., et al. Lowcomplexity portable passive drone surveillance via SDR-based signal processing. IEEE Communications Magazine, 2018, vol. 56, no. 4, p. 112-118. DOI: 10.1109/MCOM.2018.1700424

[23] XIAO, Y., ZHANG, X. Micro-UAV detection and identification based on radio frequency signature. In 2019 6th International Conference on Systems and Informatics (ICSAI). Shanghai (China), 2019, p. 1056-1062. DOI: 10.1109/ICSAI48974.2019.9010185

[24] MEDAIYESE, O. O., SYED, A., LAUF, A. P. Machine Learning Framework for RF-Based Drone Detection and Identification System. 2020, p. 1-6. [online] Available at: https://arxiv.org/ftp/arxiv/papers/2003/2003.02656.pdf

[25] KUDOH, E., FUJISAWA, H., SATOH, K. Construction of frequency-hopping system using RF communications trainer. In Proceedings of the Tenth International Conference on Ubiquitous and Future Networks (ICUFN). Prague (Czechia), Jul. 2018, p. 280-282.

[26] FU, W., HU, Z., LI, D. A sorting algorithm for multiple frequencyhopping signals in complex electromagnetic environments. Circuits Systems and Signal Processing, 2020, vol. 39, no. 1, p. 245-267. DOI: $10.1007 / \mathrm{s} 00034-019-01160-8$

[27] WU, Z., MA, X. X., ZHANG, L. Anti-jamming ability research on COFDM modulation of UAV image transmission. Advanced Materials Research, 2014, vol. 1079, p. 614-617. DOI: 10.4028/www.scientific.net/AMR.1079-1080.614

[28] JIA, Z. Design of UAV telemetry image transmission system based on H.264 standard. Automation \& Instrumentation, 2017, no. 6, p. $130-132$. 
[29] TERRIEN, J., MARQUE, C., GERMAIN, G. Ridge extraction from the time-frequency representation (TFR) of signals based on an image processing approach: Application to the analysis of uterine electromyogram AR TFR. IEEE Transactions on Biomedical Engineering, 2008, vol. 55, no. 5, p. 1496-1503. DOI: 10.1109/TBME.2008.918556

[30] PAN, J. J., ZHU, H. W., CHEN, L. S. Time-frequency ridge analysis using on distinguish regular signal from arc-fault signal. In IEEE International Workshop on Intelligent Energy Systems. Vienna (Austria), 2013, p. 203-208. DOI: 10.1109/IWIES.2013.6698586

[31] COLOMINAS, M. A., MEIGNEN, S., PHAM, D. H. Fully adaptive ridge detection based on STFT phase information. IEEE Signal Processing Letters, 2020, vol. 27, p. 620-624. DOI: 10.1109/LSP.2020.2987166

[32] TEKBIYIK, K., ALAKOCA, H., TUĞREL, H. B., et al. Identification of OFDM signals using cyclic autocorrelation function. In 2016 National Conference on Electrical, Electronics and Biomedical Engineering (ELECO). Bursa (Turkey), 2016, p. 622-626.

[33] AllahHAm, M. S., KHATTAB, T., MOHAMED A. Deep learning for RF-based drone detection and identification: A multichannel 1-D convolutional neural networks approach. In 2020 IEEE International Conference on Informatics, IoT, and Enabling Technologies (ICIoT). Doha (Qatar), 2020, p. 1-6. DOI: 10.1109/ICIoT48696.2020.9089657

\section{About the Authors ...}

Yuelei XIE was born in Handan, Hebei, China. He re- ceived his B.S. in Information and Communication Engineering from the Guilin University of Electronic Science and Technology, in 1997, and the M.S. degree from the Guilin University of Electronic Technology, China, in 2000. He joined the Guilin University of Electronic Technology in 1997, where he has been an Associate Professor, since 2013. At present, he is mainly engaged in the research of communication signal processing, ASIC design and implementation of signal processing, FPGA digital system design.

Ping JIANG (corresponding author) was born in Chengdu, Sichuan, China. He received the B.S. degree in Electronic Engineering from Chengdu Technological University, Chengdu, China, in 2017. He is currently pursuing the M.S. degree in Electronics and Communication Engineering with the Guilin University of Electronic Technology. His current research interests include UAV signal detection and recognition and FPGA digital system design.

Xiao XIAO was born in Nanyang, Henan, China. She received the B.S. degree in Electronic Information Engineering from the Institute of Information Technology, Guilin University of Electronic Technology, Guilin, China, in 2019. She is currently pursuing the M.S. degree with the Guilin University of Electronic Technology. Her current interests include signal processing and signal recognition. 